Review

\title{
Solid phase assays versus automated indirect immunofluorescence for detection of antinuclear antibodies
}

\author{
Jolien Claessens ${ }^{\mathrm{a}}$, Thibaut Belmondo ${ }^{\mathrm{b}}$, Ellen De Langhe ${ }^{\mathrm{c}}$, Rene Westhovens ${ }^{\mathrm{c}}$, Koen Poesen ${ }^{\mathrm{a}, \mathrm{d}}$, Sophie Hüe ${ }^{\mathrm{b}}$, \\ Daniel Blockmans ${ }^{\text {e,f }}$, Michael Mahler ${ }^{\mathrm{g}}$, Marvin J. Fritzler ${ }^{\mathrm{h}}$, Xavier Bossuyt ${ }^{\mathrm{a}, \mathrm{f}, *}$ \\ a Laboratory Medicine, University Hospitals Leuven, Belgium \\ b Department of Laboratory Medicine, Henri Mondor Hospital, Créteil, France \\ c Department of Rheumatology, University Hospitals Leuven, Leuven, Belgium \\ d Department of Neurosciences, KU Leuven, Leuven, Belgium \\ e Department of General Internal Medicine, University Hospitals Leuven, Leuven, Belgium \\ ${ }^{\mathrm{f}}$ Department of Microbiology and Immunology, KU Leuven, Leuven, Belgium \\ ${ }^{g}$ Research and Development, Inova Diagnostics, San Diego, USA \\ ${ }^{\text {h }}$ Cumming School of Medicine, University of Calgary, Alberta, Canada
}

\section{A R T I C L E I N F O}

\section{Article history:}

Received 27 November 2017

Accepted 3 December 2017

Available online 8 March 2018

\begin{abstract}
A B S T R A C T
Solid phase assays (SPAs) and automated microscope systems are increasingly used to screen for antinuclear antibodies (ANAs). The goal of this study was to evaluate the performance of three automated ANA screening assays; NOVA Lite HEp-2 using NOVA View ${ }^{\circledR}$ (NV, Inova Diagnostics), an automated indirect immunofluorescence method, EliA ${ }^{\mathrm{TM}}$ CTD Screen (Fluorescence Enzyme Immunoassay, FEIA; Thermo Fisher) and QUANTA Flash ${ }^{\circledR}$ CTD Screen Plus (Chemiluminescence immunoassay, CIA; Inova Diagnostics).

The assays were performed on 480 diagnostic samples from patients with an ANA-associated rheumatic disease (AARD; systemic lupus erythematosus, primary Sjögren's syndrome, systemic sclerosis, inflammatory myopathy, mixed connective tissue disease) and on 767 samples from diseased and healthy controls.

Using cut-offs proposed by the manufacturers, the sensitivity was 95\%, 80.5\% and $86 \%$ for NV, FEIA and CIA, respectively. The corresponding specificity was $61 \%$ (NV), $97.5 \%$ (FEIA) and $88 \%$ (CIA). The sensitivity associated with a specificity of $\sim 95 \%$ was $79 \%, 82 \%$ and $78 \%$ for NV, FEIA, and CIA, respectively. Receiver operating characteristics (ROC) curve analysis revealed no differences in area under the curve (AUC) between the 3 assays when all diseases were grouped. For Sjögren's syndrome, the AUC was higher for SPAs than for NV, whereas for systemic sclerosis, the AUC was higher for NV than for CIA. For all assays, the likelihood ratio for AARD increased with increasing antibody levels and for double positivity of NV with SPA.

In conclusion, the performance of automated SPA and IIF was assay- and disease-dependent. Taking into account antibody levels and combining IIF with SPA adds value.
\end{abstract}

(C) 2018 Elsevier B.V. All rights reserved.

\section{Contents}

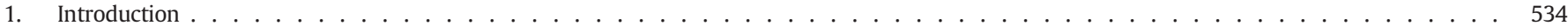

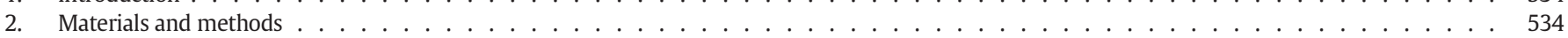

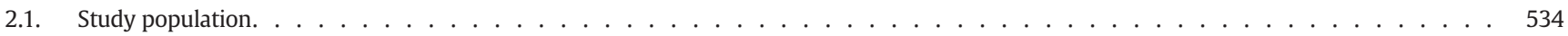

2.2. ANA screening by automated indirect immunofluorescence (NOVA View® NV $^{2} \ldots \ldots \ldots$

2.3. ANA screening by SPAs . . . . . . . . . . . . . . . . . . . . . . . . . . . . . . . . . . . . . . . . . 5535

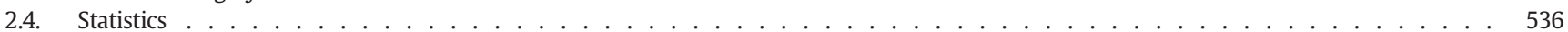

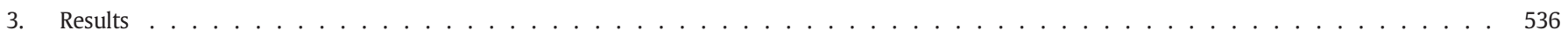

3.1. Diagnostic performance characteristics of assays . . . . . . . . . . . . . . . . . . . . . . . . . 536

3.2. SSc-related autoantibodies . . . . . . . . . . . . . . . . . . . . . . . . . . . . . . . . . . . . . . . . . . . . .

3.3. Test result-specific likelihood ratios . . . . . . . . . . . . . . . . . . . . . . . . . . . . . . . . . . 537

\footnotetext{
* Corresponding author at: Laboratory Medicine, University Hospitals Gasthuisberg, Herestraat 49, 3000 Leuven, Belgium.

E-mail address: Xavier.Bossuyt@uzleuven.be (X. Bossuyt).
} 


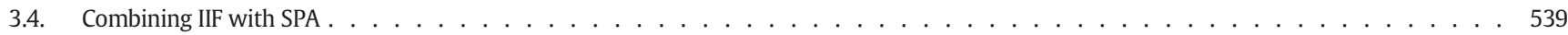

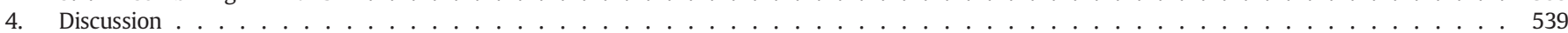

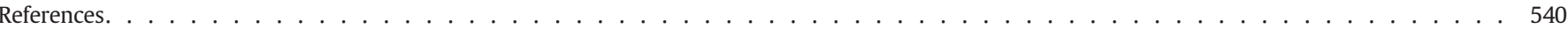

\section{Introduction}

Detection of antinuclear antibodies (ANAs) supports the clinical diagnosis of ANA-associated rheumatic diseases (AARDs), such as systemic lupus erythematosus (SLE), systemic sclerosis (SSc), primary Sjögren's syndrome ( $\mathrm{SjS}$ ), idiopathic inflammatory myopathies (IIM) and mixed connective tissue disease (MCTD). ANAs must be interpreted in light of the clinical symptoms, as they can also be found in apparently healthy individuals, in individuals with 'incomplete' or 'very early' AARD or in patients with diseases different from AARDs [1]. Indirect immunofluorescence (IIF) on human epidermoid laryngeal carcinoma cells (HEp-2 or HEp-2000 cells) is considered the "gold standard" technique for ANA screening [2]. Drawbacks of IIF are its extensive workload, lack of standardization and high false positivity in apparently healthy individuals and patients with diseases different from AARDs. To overcome these downsides, solid phase assays (SPAs) have been developed for screening the most commonly detected ANAs in AARD [2]. Studies comparing SPAs with IIF reported a higher specificity, but slightly lower sensitivity for SPAs [3]. Comparing SPAs among each other, however, indicated variable assay sensitivity and specificity, explained by differences in analyte characteristics and cut-off levels [4]. Even though SPAs can be automated and offer objective results, IIF remains valuable for the detection of less frequent antibodies not detected by SPAs. Moreover, diagnostic criteria for AARD diagnosis were developed based on IIF results [5]. Therefore, efforts have been made to automate and standardize IIF.

Digital microscopy with image interpretation is an important step towards more objective IIF results. Computerized image interpretation includes quantification of fluorescence intensity and identification of the fluorescence pattern. In some cases, the fluorescence pattern has a diagnostic value (e.g. centromere pattern associated with limited SSc) and can give an indication of the antibody specificity [2]. Quantification of fluorescence intensity is valuable as higher fluorescence intensities are related to higher likelihoods for AARD [6-8].

The present study evaluated the performance characteristics of three automated assays for ANA screening: (i) an automated IIF method
(NOVA Lite HEp-2 on NOVA View®, NV, Inova Diagnostics), (ii) a fluorescence enzyme immunoassay (FEIA, EliA ${ }^{\mathrm{TM}}$ CTD screen, Phadia, ThermoFisher) and (iii) a chemiluminescent immunoassay (CIA, QUANTA Flash ${ }^{\circledR}$ CTD screen Plus, INOVA Diagnostics). FEIA and CIA differ from each other in antigen composition, assay methodology and detection technique.

\section{Materials and methods}

\subsection{Study population}

Assays were evaluated on samples obtained from patients diagnosed with an AARD $(\mathrm{n}=480)$ and diseased and healthy controls $(\mathrm{n}=767)$. AARD patients were classified as SLE $[\mathrm{n}=119$; male/female 18/101; median age (range) 32.5 (15-72)], SSc $[\mathrm{n}=220$; male/female 55/165; median age (range) 45 (18-85)], SjS $[\mathrm{n}=65$; male/female 10/55; median age (range) 49 (16-89)], IIM [ $\mathrm{n}=50$; male/female 19/31; median age (range) 50.5 (24-84)], and MCTD $[\mathrm{n}=26$; male/female 3/23; median age (range) 32 (16-66)] according to the respective classification or diagnostic criteria [9-13]. Samples from patients not fulfilling the classification or diagnostic criteria or those receiving immunosuppressive therapy were excluded. All samples were obtained at the time of diagnosis or within 3 months after diagnosis.

Diseased controls included: (i) consecutive patients for whom ANA screening was requested by a rheumatologist or specialist in general internal medicine but for whom afterwards AARD was excluded (DC) [n $=314 ;$ male/female 76/238; median age (range) 43 (16-90)], (ii) patients suffering from chronic fatigue syndrome (CFS) $[\mathrm{n}=150$; male/female 28/122; median age (range) 41 (16-75)], (iii) patients with polymyalgia rheumatica $[\mathrm{n}=6$; male/female $2 / 4$; median age (range) $71(58-83)]$ and rheumatoid arthritis $[\mathrm{n}=18$; male/female 6/12; median age (range) 48 (18-76)]. Healthy blood donors were included as well (BD) [n = 279; male/female 146/133; median age (range) 46 (18-69)].
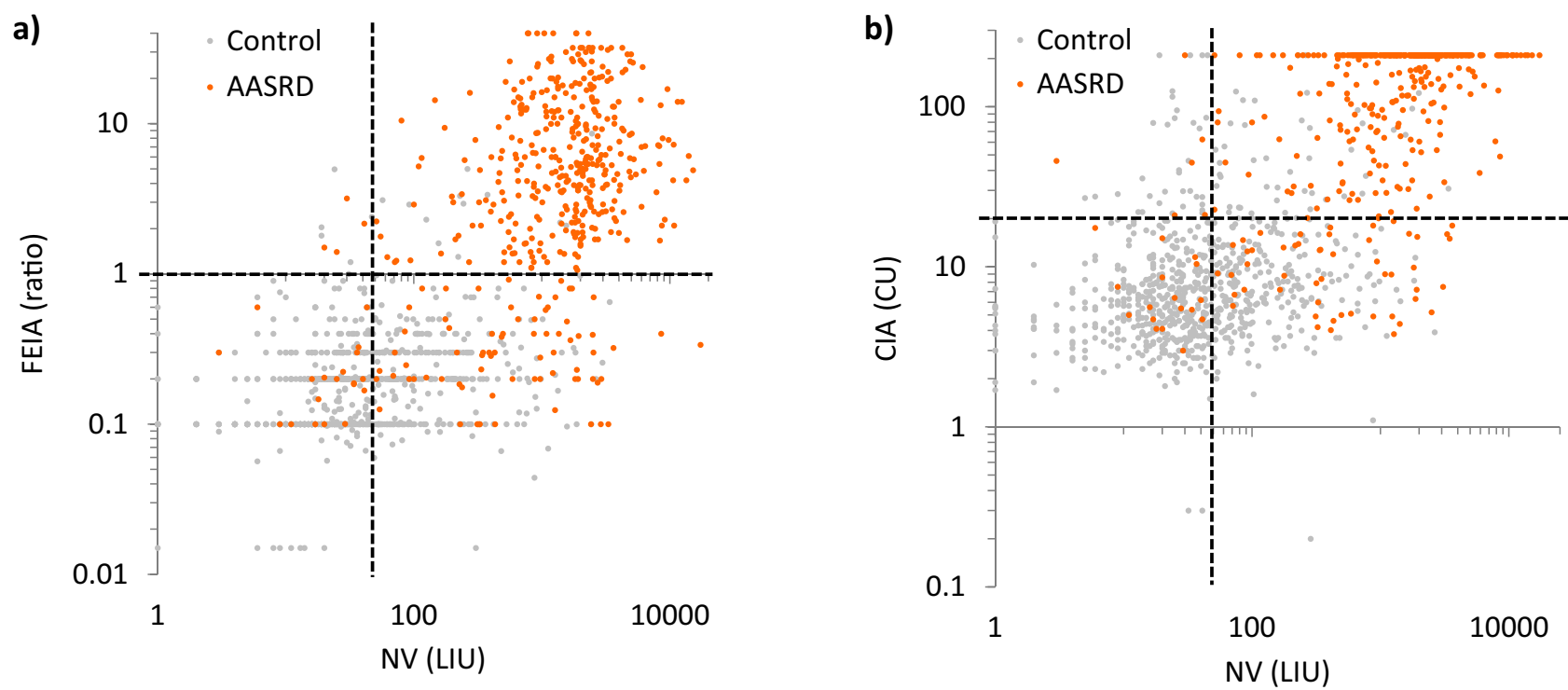

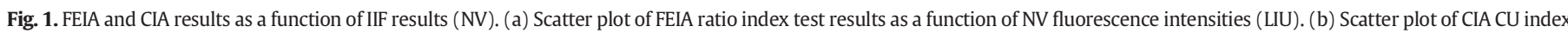
test results as a function of NV fluorescence intensities (LIU). Dashed lines indicate cut-offs for positivity suggested by the manufacturers. Red dots: AARD; grey dots: controls. 
Samples were from three different locations [University Hospitals Leuven, Belgium; Hôpital Henri Mondor, Créteil, France; Mitogen Advanced Diagnostics, Cumming School of Medicine, University of Calgary, Alberta, Canada]. The Hôpital Henri Mondor provided a total of 115 samples obtained from patients diagnosed with SLE $(n=13)$, SSc ( $n$ $=11), \operatorname{SjS}(n=3), \operatorname{PM} / \mathrm{DM}(\mathrm{n}=8), \operatorname{MCTD}(\mathrm{n}=1)$ and samples of diseased controls $(n=79)$. The Mitogen Advanced Diagnostics provided 67 samples of patients diagnosed with SSc. The samples were collected from 2007 till 2015 and stored at $-80{ }^{\circ} \mathrm{C}$ until analysis. Analyses were performed at the Department of Laboratory Medicine of the University Hospitals Leuven, Belgium. The study was approved by the ethics committee of the University Hospital of Leuven (ML6225) and complied with the Helsinki Declaration.

\subsection{ANA screening by automated indirect immunofluorescence (NOVA View $\left.{ }^{\circledR}, N V\right)$}

Samples were diluted for IIF using a QUANTA Lyser® 2 (Inova Diagnostics, San Deigo, CA, USA). IIF was performed with the NOVA Lite ${ }$ HEp-2 ANA kit (Inova Diagnostics) as substrate using goat anti-human IgG specific fluorescein (FITC)-labeled conjugate containing 4'-6diamidino-2-phenylindol (DAPI) [8]. Automated IIF was performed with a NV instrument (Inova Diagnostics) (software version 2.0.3.). Positivity of the samples was based on the fluorescence intensity (light intensity units, LIU) at a serum dilution of $1 / 80$ (recommended by manufacturer), the cut-off for positivity proposed by the manufacturer being $>48 \mathrm{LIU}$. The specific fluorescence patterns were not included in the assessment of NV performance [14].

\subsection{ANA screening by SPAs}

The Phadia EliA ${ }^{\text {TM }}$ CTD screen assay (FEIA, ThermoFisher) was performed according to the instructions of the manufacturer on a Unicap 250 instrument (Phadia, Thermo-Fisher). The cut-off ratio for positivity proposed by the manufacturer was $>1.00$ ratio. The manufacturer states that test results between 0.70 and 1.00 are inconclusive and such results were considered negative when assessing assay performance. The antigens included are ds-DNA, Sm, SSA/Ro60 and Ro52/TRIM21, SS-B/La, Centromere B, Scl-70, Mi-2, RNA-Pol III, PM/Scl, Jo-1, PCNA, Ribosomal-P protein, U1-RNP (RNP-70, A, C), and fibrillarin.

The QUANTA Flash ${ }^{\circledR}$ CTD screen Plus assay (CIA, Inova Diagnostics) was performed according to the instructions of the manufacturer on a BIO-FLASH ${ }^{\circledR}$ instrument fitted with a luminometer (Inova Diagnostics). The cut-off for assay positivity proposed by the manufacturer was $\geq 20$

Table

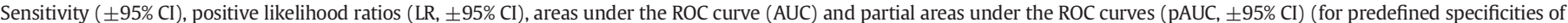

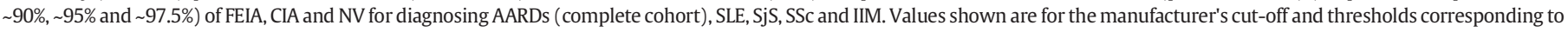
specificities of $\sim 90.0 \%, \sim 95.0 \%$ and $\sim 97.5 \%$.

\begin{tabular}{|c|c|c|c|c|c|c|c|c|c|c|}
\hline & & \multicolumn{3}{|c|}{ Sensitivity $( \pm 95 \% \mathrm{CI}) \%$} & \multicolumn{3}{|l|}{$\mathrm{LR}( \pm 95 \% \mathrm{CI})$} & \multicolumn{3}{|c|}{ AUC/pAUC $( \pm 95 \% \mathrm{CI}) \%$} \\
\hline & & FEIA & $\mathrm{CIA}$ & NV & FEIA & CIA & NV & FEIA & $\mathrm{CIA}$ & NV \\
\hline \multirow[t]{5}{*}{ Cut-off manufacturers } & AARD & $\begin{array}{l}80.5 \\
(76.7-83.8)\end{array}$ & $\begin{array}{l}85.9 \\
(82.5-88.7)\end{array}$ & $\begin{array}{l}95.2 \\
(92.9-96.8)\end{array}$ & $\begin{array}{l}32.5 \\
(20.8-50.8)\end{array}$ & $\begin{array}{l}7.3 \\
(6.0-9.0)\end{array}$ & $\begin{array}{l}2.4 \\
(2.2-2.7)\end{array}$ & $\begin{array}{l}0.931 \\
(0.916-0.947)\end{array}$ & $\begin{array}{l}0.926 \\
(0.910-0.942)\end{array}$ & $\begin{array}{l}0.937 \\
(0.922-0.952)\end{array}$ \\
\hline & SLE & $\begin{array}{l}79.8 \\
(71.5-86.6)\end{array}$ & $\begin{array}{l}85.7 \\
(78.1-91.5)\end{array}$ & $\begin{array}{l}93.8 \\
(85.0-98.3)\end{array}$ & $\begin{array}{l}32.2 \\
(20.5-50.7)\end{array}$ & $\begin{array}{l}7.3 \\
(5.9-9.0)\end{array}$ & $\begin{array}{l}2.4 \\
(2.2-2.7)\end{array}$ & $\begin{array}{l}0.947 \\
(0.924-0.970)\end{array}$ & $\begin{array}{l}0.933 \\
(0.906-0.959)\end{array}$ & $\begin{array}{l}0.941 \\
(0.916-0.966)\end{array}$ \\
\hline & $\mathrm{SSc}$ & $\begin{array}{l}82.3 \\
(76.6-87.1)\end{array}$ & $\begin{array}{l}87.3 \\
(82.1-91.4)\end{array}$ & $\begin{array}{l}97.7 \\
(94.8-99.3)\end{array}$ & $\begin{array}{l}33.2 \\
(21.2-52.0)\end{array}$ & $\begin{array}{l}7.4 \\
(6.1-9.1)\end{array}$ & $\begin{array}{l}2.5 \\
(2.3-2.7)\end{array}$ & $\begin{array}{l}0.940 \\
(0.919-0.961)\end{array}$ & $\begin{array}{l}0.931^{\mathrm{b}} \\
(0.910-0.953)\end{array}$ & $\begin{array}{l}0.961^{\mathrm{b}} \\
(0.946-0.976)\end{array}$ \\
\hline & SjS & $\begin{array}{l}91.0 \\
(81.5-96.6)\end{array}$ & $\begin{array}{l}91.0 \\
(81.5-96.6)\end{array}$ & $\begin{array}{l}94.1 \\
(88.3-97.6)\end{array}$ & $\begin{array}{l}36.8 \\
(23.4-57.7)\end{array}$ & $\begin{array}{l}7.8 \\
(6.3-9.6)\end{array}$ & $\begin{array}{l}2.4 \\
(2.2-2.7)\end{array}$ & $\begin{array}{l}0.959^{\mathrm{a}} \\
(0.926-0.992)\end{array}$ & $\begin{array}{l}0.949^{\mathrm{b}} \\
(0.916-0.982)\end{array}$ & $\begin{array}{l}0.926^{\mathrm{a}, \mathrm{b}} \\
(0.883-0.969)\end{array}$ \\
\hline & IIM & $\begin{array}{l}50.0 \\
(35.5-64.5)\end{array}$ & $\begin{array}{l}66.0 \\
(51.2-78.8)\end{array}$ & $\begin{array}{l}86.0 \\
(73.3-94.2)\end{array}$ & $\begin{array}{l}20.2 \\
(12.0-34.1)\end{array}$ & $\begin{array}{l}5.6 \\
(4.3-7.4)\end{array}$ & $\begin{array}{l}2.2 \\
(1.9-2.5)\end{array}$ & $\begin{array}{l}0.786 \\
(0.708-0.846)\end{array}$ & $\begin{array}{l}0.827 \\
(0.755-0.900)\end{array}$ & $\begin{array}{l}0.814 \\
(0.744-0.884)\end{array}$ \\
\hline \multirow[t]{5}{*}{ 90.0\% spec. } & AARD & $\begin{array}{l}84.9 \\
(81.4-87.8)\end{array}$ & $\begin{array}{l}84.6 \\
(81.1-87.6)\end{array}$ & $\begin{array}{l}87.3 \\
(84.0-90.0)\end{array}$ & $\begin{array}{l}8.5 \\
(6.9-10.7)\end{array}$ & $\begin{array}{l}8.5 \\
(6.9-10.7)\end{array}$ & $\begin{array}{l}8.8 \\
(7.2-11.1)\end{array}$ & $\begin{array}{l}0.077^{\mathrm{c}} \\
(0.072-0.082)\end{array}$ & $\begin{array}{l}0.070^{c} \\
(0.065-0.076)\end{array}$ & $\begin{array}{l}0.072 \\
(0.065-0.078)\end{array}$ \\
\hline & SLE & $\begin{array}{l}84.9 \\
(77.2-90.3)\end{array}$ & $\begin{array}{l}83.2 \\
(75.3-88.9)\end{array}$ & $\begin{array}{l}89.9 \\
(83.0-94.2)\end{array}$ & $\begin{array}{l}8.5 \\
(6.8-10.7)\end{array}$ & $\begin{array}{l}8.3 \\
(6.7-10.6)\end{array}$ & $\begin{array}{l}9.1 \\
(7.4-11.5)\end{array}$ & $\begin{array}{l}0.077^{\mathrm{c}} \\
(0.070-0.084)\end{array}$ & $\begin{array}{l}0.068^{\mathrm{c}} \\
(0.059-0.077)\end{array}$ & $\begin{array}{l}0.073 \\
(0.066-0.081)\end{array}$ \\
\hline & SSc & $\begin{array}{l}87.3 \\
(82.1-91.1)\end{array}$ & $\begin{array}{l}86.8 \\
(81.6-90.7)\end{array}$ & $\begin{array}{l}91.4 \\
(86.8-94.4)\end{array}$ & $\begin{array}{l}8.7 \\
(7.1-11.0)\end{array}$ & $\begin{array}{l}8.7 \\
(7.0-10.9)\end{array}$ & $\begin{array}{l}9.2 \\
(7.5-11.7)\end{array}$ & $\begin{array}{l}0.078^{c} \\
(0.073-0.084)\end{array}$ & $\begin{array}{l}0.071^{\mathrm{b}, \mathrm{c}} \\
(0.064-0.077)\end{array}$ & $\begin{array}{l}0.079^{\mathrm{b}} \\
(0.072-0.084)\end{array}$ \\
\hline & SjS & $\begin{array}{l}91.0 \\
(81.4-96.1)\end{array}$ & $\begin{array}{l}91.0 \\
(81.4-96.1)\end{array}$ & $\begin{array}{l}89.2 \\
(79.0-94.9)\end{array}$ & $\begin{array}{l}9.1 \\
(7.3-11.5)\end{array}$ & $\begin{array}{l}9.1 \\
(7.3-11.5)\end{array}$ & $\begin{array}{l}8.7 \\
(7.0-11.1)\end{array}$ & $\begin{array}{l}0.090^{\mathrm{a}, \mathrm{c}} \\
(0.084-0.096)\end{array}$ & $\begin{array}{l}0.081^{\mathrm{b}, \mathrm{c}} \\
(0.072-0.090)\end{array}$ & $\begin{array}{l}0.069^{\mathrm{a}, \mathrm{b}} \\
(0.060-0.077)\end{array}$ \\
\hline & IIM & $\begin{array}{l}58.0 \\
(44.2-70.6)\end{array}$ & $\begin{array}{l}64.0 \\
(50.1-75.8)\end{array}$ & $\begin{array}{l}56.0 \\
(42.3-68.8)\end{array}$ & $\begin{array}{l}5.8 \\
(4.1-7.9)\end{array}$ & $\begin{array}{l}6.4 \\
(4.7-8.6)\end{array}$ & $\begin{array}{l}5.7 \\
(4.0-7.8)\end{array}$ & $\begin{array}{l}0.048 \\
(0.036-0.060)\end{array}$ & $\begin{array}{l}0.050^{\mathrm{b}} \\
(0.039-0.063)\end{array}$ & $\begin{array}{l}0.036^{\mathrm{b}} \\
(0.026-0.049)\end{array}$ \\
\hline \multirow[t]{5}{*}{ 95.0\% spec. } & AARD & $\begin{array}{l}82.2 \\
(78.5-85.3)\end{array}$ & $\begin{array}{l}77.6 \\
(73.6-81.1)\end{array}$ & $\begin{array}{l}79.0 \\
(75.3-82.6)\end{array}$ & $\begin{array}{l}17.5 \\
(13.1-25.2)\end{array}$ & $\begin{array}{l}15.7 \\
(11.4-21.4)\end{array}$ & $\begin{array}{l}16.0 \\
(11.7-21.9)\end{array}$ & $\begin{array}{l}0.035^{\mathrm{a}, \mathrm{c}} \\
(0.033-0.039)\end{array}$ & $\begin{array}{l}0.029^{c} \\
(0.025-0.034)\end{array}$ & $\begin{array}{l}0.029^{\mathrm{a}} \\
(0.025-0.034)\end{array}$ \\
\hline & SLE & $\begin{array}{l}80.7 \\
(72.6-86.8)\end{array}$ & $\begin{array}{l}73.1 \\
(64.5-80.3)\end{array}$ & $\begin{array}{l}82.4 \\
(74.4-88.2)\end{array}$ & $\begin{array}{l}17.2 \\
(12.6-24.8)\end{array}$ & $\begin{array}{l}14.8 \\
(10.9-21.3)\end{array}$ & $\begin{array}{l}16.6 \\
(12.3-23.6)\end{array}$ & $\begin{array}{l}0.036^{\mathrm{a}, \mathrm{c}} \\
(0.032-0.040)\end{array}$ & $\begin{array}{l}0.027^{c} \\
(0.022-0.032)\end{array}$ & $\begin{array}{l}0.028^{\mathrm{a}} \\
(0.023-0.035)\end{array}$ \\
\hline & $\mathrm{SSc}$ & $\begin{array}{l}85.0 \\
(79.6-89.1)\end{array}$ & $\begin{array}{l}79.5 \\
(73.7-84.4)\end{array}$ & $\begin{array}{l}85.0 \\
(79.6-89.1)\end{array}$ & $\begin{array}{l}18.1 \\
(13.4-25.9)\end{array}$ & $\begin{array}{l}16.1 \\
(12.0-23.0)\end{array}$ & $\begin{array}{l}17.2 \\
(12.8-24.2)\end{array}$ & $\begin{array}{l}0.035^{c} \\
(0.030-0.040)\end{array}$ & $\begin{array}{l}0.029^{c} \\
(0.024-0.033)\end{array}$ & $\begin{array}{l}0.034 \\
(0.029-0.039)\end{array}$ \\
\hline & SjS & $\begin{array}{l}91.0 \\
(81.4-96.1)\end{array}$ & $\begin{array}{l}89.6 \\
(79.6-95.1)\end{array}$ & $\begin{array}{l}80.0 \\
(68.5-88.0)\end{array}$ & $\begin{array}{l}19.4 \\
(14.2-27.6)\end{array}$ & $\begin{array}{l}18.1 \\
(13.4-25.8)\end{array}$ & $\begin{array}{l}15.7 \\
(11.4-22.5)\end{array}$ & $\begin{array}{l}0.044^{\mathrm{a}, \mathrm{c}} \\
(0.040-0.047)\end{array}$ & $\begin{array}{l}0.037^{\mathrm{b}, \mathrm{c}} \\
(0.032-0.042)\end{array}$ & $\begin{array}{l}0.026^{\mathrm{a}, \mathrm{b}} \\
(0.019-0.033)\end{array}$ \\
\hline & IIM & $\begin{array}{l}52.0 \\
(38.5-65.2)\end{array}$ & $\begin{array}{l}56.0 \\
(42.3-68.8)\end{array}$ & $\begin{array}{l}40.0 \\
(27.6-53.8)\end{array}$ & $\begin{array}{l}11.1 \\
(7.2-17.0)\end{array}$ & $\begin{array}{l}11.3 \\
(7.6-17.1)\end{array}$ & $\begin{array}{l}8.1 \\
(4.9-12.8)\end{array}$ & $\begin{array}{l}0.020^{\mathrm{a}} \\
(0.014-0.027)\end{array}$ & $\begin{array}{l}0.020^{\mathrm{b}} \\
(0.013-0.027)\end{array}$ & $\begin{array}{l}0.011^{\mathrm{a}, \mathrm{b}} \\
(0.0066-0.017)\end{array}$ \\
\hline \multirow[t]{5}{*}{ 97.5\% spec. } & AARD & $\begin{array}{l}80.5 \\
(76.7-83.9)\end{array}$ & $\begin{array}{l}69.5 \\
(65.2-73.4)\end{array}$ & $\begin{array}{l}66.3 \\
(61.9-70.3)\end{array}$ & $\begin{array}{l}32.5 \\
(20.8-50.8)\end{array}$ & $\begin{array}{l}28.1 \\
(17.9-43.9)\end{array}$ & $\begin{array}{l}26.7 \\
(17.1-41.9)\end{array}$ & $\begin{array}{l}0.015^{\mathrm{a}, \mathrm{c}} \\
(0.013-0.018)\end{array}$ & $\begin{array}{l}0.011^{\mathrm{c}} \\
(0.009-0.015)\end{array}$ & $\begin{array}{l}0.011^{\mathrm{a}} \\
(0.008-0.013)\end{array}$ \\
\hline & SLE & $\begin{array}{l}79.8 \\
(71.6-86.1)\end{array}$ & $\begin{array}{l}63.0 \\
(54.1-71.2)\end{array}$ & $\begin{array}{l}62.2 \\
(53.2-70.4)\end{array}$ & $\begin{array}{l}32.2 \\
(21.3-54.4)\end{array}$ & $\begin{array}{l}25.4 \\
(16.5-43.1)\end{array}$ & $\begin{array}{l}25.1 \\
(16.3-42.8)\end{array}$ & $\begin{array}{l}0.016^{\mathrm{a}, \mathrm{c}} \\
(0.014-0.018)\end{array}$ & $\begin{array}{l}0.011^{\mathrm{c}} \\
(0.0079-0.014)\end{array}$ & $\begin{array}{l}0.010^{\mathrm{a}} \\
(0.0068-0.014)\end{array}$ \\
\hline & SSc & $\begin{array}{l}82.3 \\
(76.6-86.8)\end{array}$ & $\begin{array}{l}70.5 \\
(64.1-76.1)\end{array}$ & $\begin{array}{l}77.7 \\
(71.7-82.7)\end{array}$ & $\begin{array}{l}33.2 \\
(22.2-56.1)\end{array}$ & $\begin{array}{l}28.4 \\
(18.8-47.9)\end{array}$ & $\begin{array}{l}31.4 \\
(20.9-52.8)\end{array}$ & $\begin{array}{l}0.014 \\
(0.0096-0.018)\end{array}$ & $\begin{array}{l}0.011 \\
(0.0073-0.014)\end{array}$ & $\begin{array}{l}0.013 \\
(0.011-0.016)\end{array}$ \\
\hline & SjS & $\begin{array}{l}91.0 \\
(81.4-96.1)\end{array}$ & $\begin{array}{l}86.6 \\
(76.1-92.9)\end{array}$ & $\begin{array}{l}61.5 \\
(49.4-72.4)\end{array}$ & $\begin{array}{l}36.8 \\
(24.3-61.5)\end{array}$ & $\begin{array}{l}35.0 \\
(23.1-59.1)\end{array}$ & $\begin{array}{l}24.1 \\
(15.4-42.4)\end{array}$ & $\begin{array}{l}0.021^{\mathrm{a}, \mathrm{c}} \\
(0.019-0.023)\end{array}$ & $\begin{array}{l}0.015^{\mathrm{b}, \mathrm{c}} \\
(0.011-0.018)\end{array}$ & $\begin{array}{l}0.0075^{\mathrm{a}, \mathrm{b}} \\
(0.0044-0.012)\end{array}$ \\
\hline & IIM & $\begin{array}{l}50.0 \\
(36.7-63.3)\end{array}$ & $\begin{array}{l}46.0 \\
(33.0-59.6)\end{array}$ & $\begin{array}{l}22.0 \\
(12.7-35.5)\end{array}$ & $\begin{array}{l}20.2 \\
(11.9-35.5)\end{array}$ & $\begin{array}{l}18.6 \\
(10.9-33.6)\end{array}$ & $\begin{array}{l}8.9 \\
(4.0-17.7)\end{array}$ & $\begin{array}{l}0.0077^{\mathrm{a}} \\
(0.0042-0.010)\end{array}$ & $\begin{array}{l}0.0072 \\
(0.0049-0.010)\end{array}$ & $\begin{array}{l}0.0038^{\mathrm{a}} \\
(0.0017-0.0071)\end{array}$ \\
\hline
\end{tabular}

\footnotetext{
Significant difference NV vs. FEIA

b Significant difference NV vs. CIA.

c Significant difference FEIA vs. CIA
} 


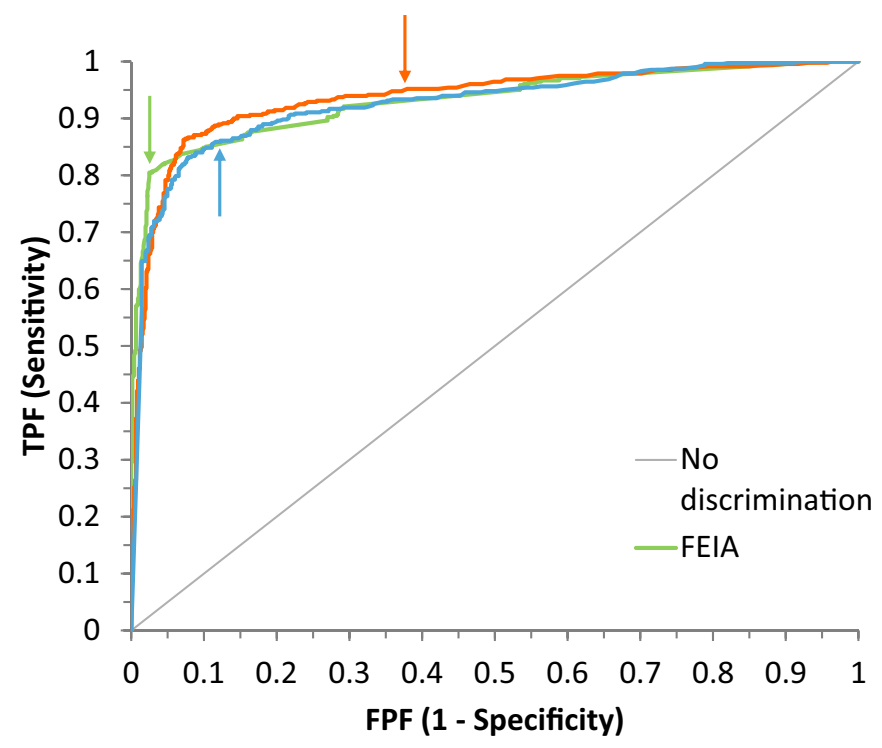

Fig. 2. Receiver operating characteristics (ROC) curves of FEIA, NV and CIA assays for diagnosing AARDs. Arrows indicate cut-off values proposed by the manufacturer.

CU. The antigens included are dsDNA, Sm/RNP, Ro60, Ro52, SS-B/La, centromere, Scl-70, Mi-2, RNA-Pol III, PM/Scl, Jo-1, PCNA, Ribosomal-P protein, $\mathrm{Ku}$, and $\mathrm{Th} / \mathrm{To}$.

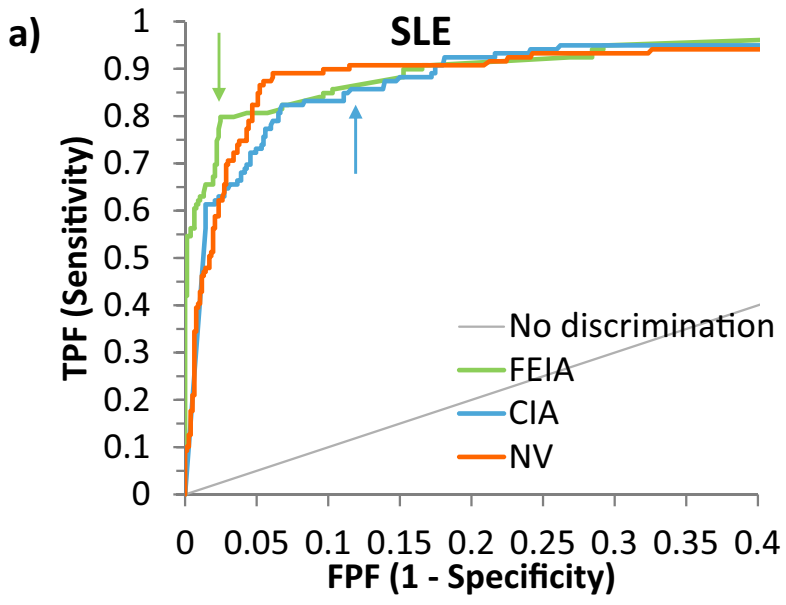

c)

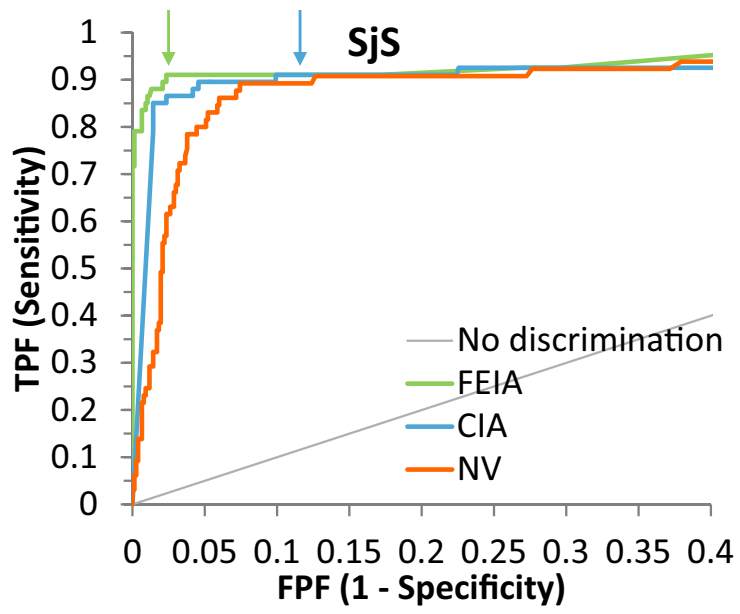

\subsection{Statistics}

Statistical analysis (ROC curve analysis, sensitivity, specificity, likelihood ratio and Odds ratio) was performed using XL Stat, Analyse-it and $\mathrm{R}$ (partial AUC). $p$-Values $<0.05$ indicated statistical significance.

\section{Results}

\subsection{Diagnostic performance characteristics of assays}

Three assays for ANA screening [automated IIF (NV), FEIA and CIA] were evaluated on 767 control samples and 480 diagnostic samples from AARD patients. The results are displayed in Fig. 1 and summarized in Table 1. With NV, a positive test was found in $39 \%$ of the controls $(47 \%$ in diseased controls, $31 \%$ in chronic fatigue syndrome and $34 \%$ in blood donors), compared to $2.5 \%$ ( $3 \%$ in diseased controls and chronic fatigue syndrome and $1 \%$ in blood donors) with FEIA and $12 \%$ (17\% in diseased controls, $9 \%$ in chronic fatigue syndrome and $6 \%$ in blood donors) with CIA, corresponding to a specificity (95\% CI) of $61.0 \%(57.5 \%-64 \%)$, 97.5\% (96.1\%-98.4\%) and 88.3\% (85.5\%-90.4\%) for NV, FEIA and CIA, respectively. Sensitivity (95\% CI) was 95.2\% (92.9\%-96.8\%), 80.5\% (76.6\%$83.8 \%$ ) and $85.9 \%(82.5 \%-88.7 \%)$ for NV, FEIA and CIA, respectively. The likelihood ratio (LR) for a positive result was $2.4,32.5$ and 7.3 and the LR for a negative result was $0.08,0.2$, and 0.2 for NV, FEIA and CIA, respectively. The odds ratio (OR) was $30.8,163.5$ and 46.8 , respectively. When thresholds were used that corresponded to pre-defined specificities

b)

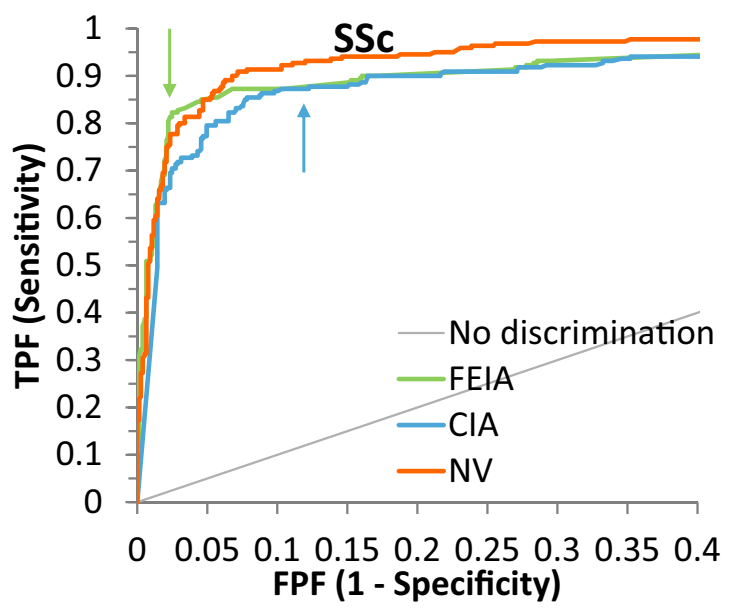

d)

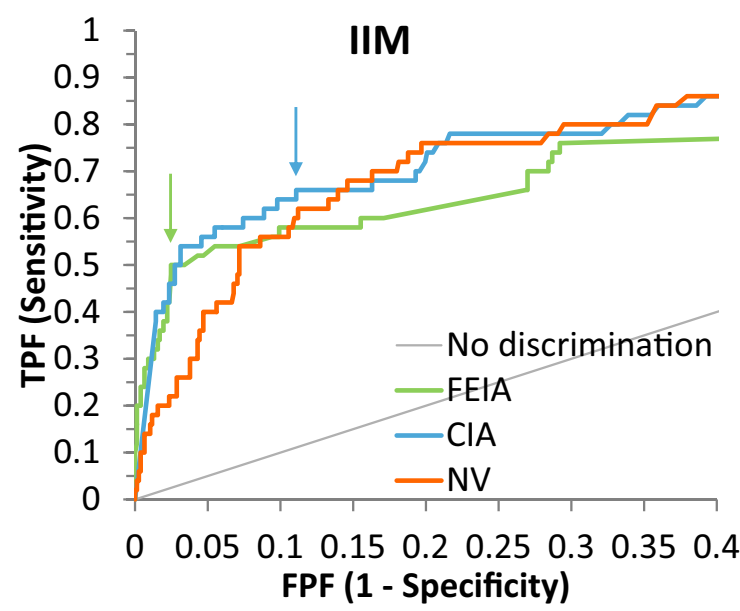

Fig. 3. Receiver operating characteristics (ROC) curves of FEIA, NV and CIA assays for SLE, SSc, SjS and IIM. Arrows indicate cut-off values proposed by the manufacturer. 
( $90 \%, \sim 95 \%, \sim 97.5 \%$ ), then the LRs and OR were more similar among assays (Table 1 ).

The sensitivities and LRs [including sensitivities and LRs corresponding to specificities of $\sim 90 \%, \sim 95 \%$ and $\sim 97.5 \%$ ] for each of the separate AARDs are summarized in Table 1.

When all AARD patients were combined, then the area under the curve (AUC) of the receiver operating characteristics (ROC) curve was not significantly different between the three assays $(p>0.05)$ (Fig. 2). However, when ROC curve analysis was performed for each disease separately, then a statistically higher AUC was observed (i) for CIA and FEIA compared to NV for SjS and (ii) for NV compared to CIA for SSc (Fig. 3). No statistically significant differences in AUC between assays were observed for SLE and IIM. The partial AUC (pAUC, calculated for specificity thresholds of $\sim 90 \%, \sim 95 \%$ and $\sim 97.5 \%$ ) was higher for the two SPAs than for NV in SjS, and in IIM for the $~ 95 \%$ specificity threshold. The pAUC at specificity thresholds $\geq 90 \%$ was higher for FEIA than for CIA when all AARD were grouped and for SLE, SjS and SSc (except for the $\sim 97.5 \%$ threshold) (Table 1).

\subsection{SSc-related autoantibodies}

FEIA and CIA assays contain different SSc-related autoantigens; fibrillarin in FEIA and Th/To and Ku in CIA. CENPB, RNA polymerase III,
$\mathrm{PM} / \mathrm{Scl}$ and Scl-70 are included in both assays. The target antigen of the antibodies was determined in samples from SSc patients that reacted positive with one of the 2 SPAs (FEIA or CIA) but negative with the other. Two samples that tested positive by FEIA and negative by CIA contained anti-fibrillarin antibodies. Thirteen samples that tested positive by CIA and negative by FEIA contained anti-Th/To antibodies ( $\mathrm{n}$ $=3$ ), anti-Ku antibodies ( $\mathrm{n}=1$ ), anti-RNA polymerase III antibodies ( $\mathrm{n}$ $=2$ ), anti-SSA/Ro60 antibodies $(\mathrm{n}=1)$ and anti-centromere antibodies $(\mathrm{n}=1)$. In 5 of these 13 patients, the target antigen could not be identified.

\subsection{Test result-specific likelihood ratios}

Thresholds were defined based on the manufacturer's cut-off and on predefined specificities ( $85 \%, \sim 95 \%, \sim 97.5 \%$ and $100 \%$ ). Based on these thresholds, test result intervals were defined and interval-specific LRs calculated. The results are shown in Fig. 4. Fig. 5 shows the test resultinterval post-test probabilities [15]

For NV, a light intensity unit (LIU) of 48 (cut-off manufacturer), 158, 522,910 , and 3382 , corresponded to a specificity of $61.0 \%, \sim 85 \%, \sim 95 \%$, $\sim 97.5 \%$ and $100 \%$, respectively. The LR $(95 \% \mathrm{CI})$ for AARD was 0.078 (0.052-0.117), 0.199 (0.131-0.302), 1.135 (0.817-1.579), 5.214 (3.159-8.607), 22.28 (14.004-35.454) and $\infty(37.8-\infty)$ for test results
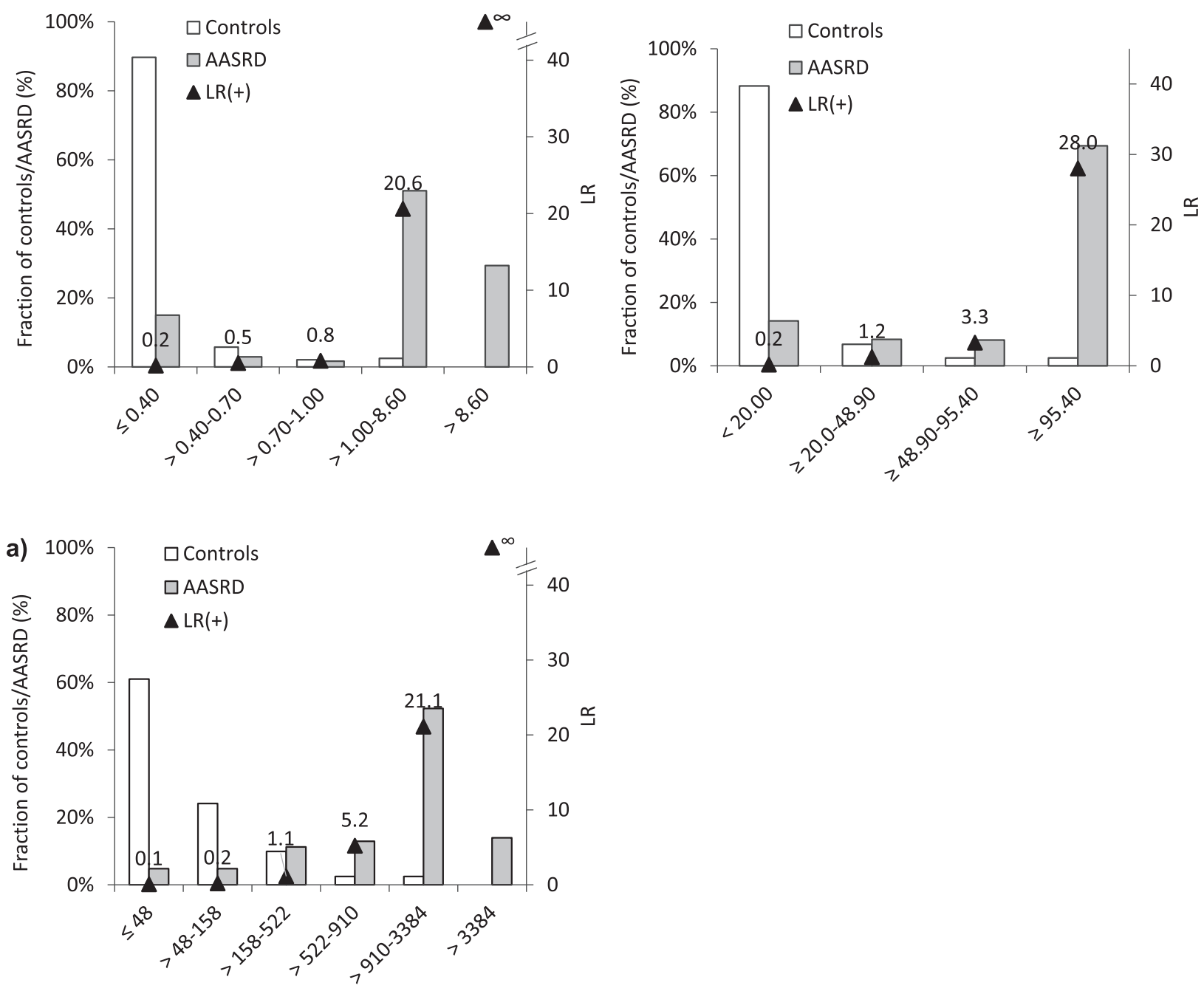

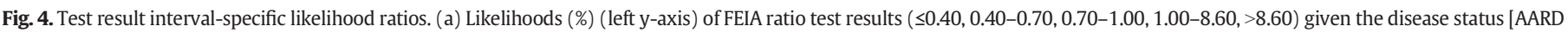

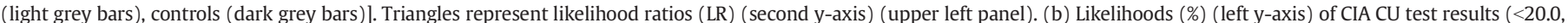

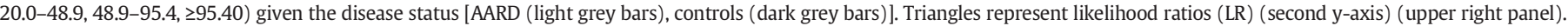

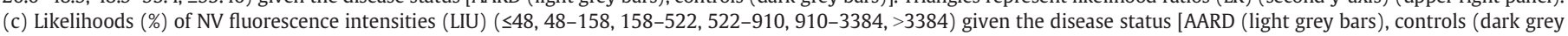
bars)]. Triangles represent likelihood ratios (LR) (second y-axis) (lower panel). 

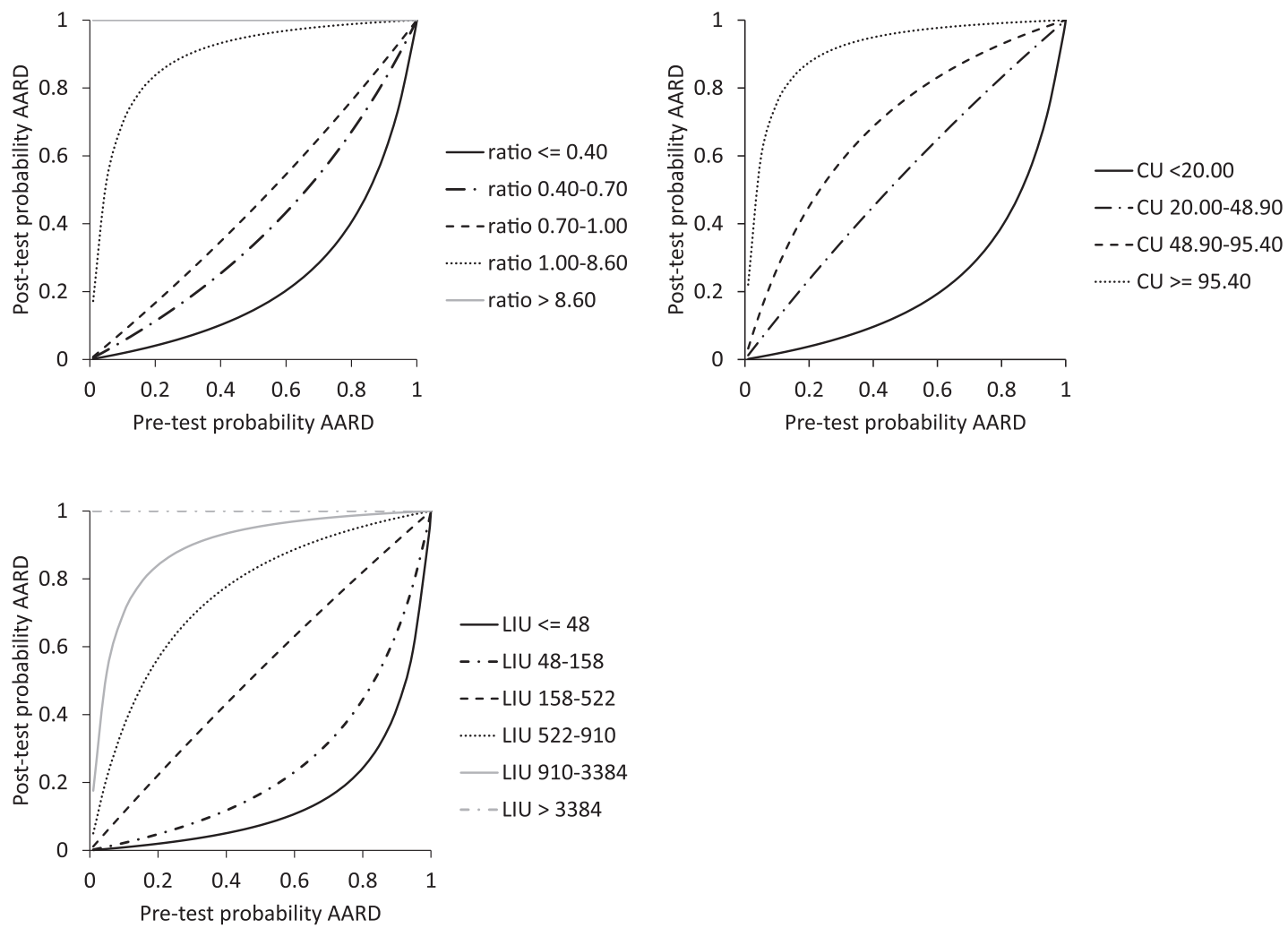

Fig. 5. Post-test probability of AARD as a function of pre-test probability and of a specific test result. Data are given for FEIA (upper left panel), CIA (upper right panel) and IIF (lower panel).

(LIU) $\leq 48,48-158,158-522,522-910,910-3382$ and $>3382$, respectively. The proportion of patients with a test result in each of these test result intervals was $5 \%, 5 \%, 11 \%, 13 \%, 52 \%$, and $14 \%$, respectively. Thus, for values $>522$ LIU, the LR for AARD was $>1 ; 79 \%$ of the AARD patients had such test result. $66 \%$ of patients had a test result with a $L R>20$ (910 LIU).

For FEIA, a ratio of $0.4,0.7,1$ (cut-off manufacturer) and 8.6 corresponded to a specificity of $\sim 85 \%, \sim 95 \%, \sim 97.5 \%$, and $100 \%$, respectively. The LR (95\% CI) for AARD was 0.167 (0.135-0.207), 0.508 (0.282-0.918), 0.752 (0.327-1.729), 20.6 (13.104-32.399) and $\infty$ $(28.112-\infty)$ for test results (ratio) $\leq 0.40,>0.40-0.70,>0.70-1.00$, $>1.00-8.60$ and $>8.60$, respectively. The fraction of patients with a test result in each of these test result intervals was $15 \%, 3 \%, 2 \%, 51 \%$, and $29 \%$, respectively. Thus, for values $>1$ (ratio), the LR for AARD was $>1$ (even $>20$ ); $80 \%$ of the AARD patients had such test result.

For CIA, a CU of 20 (cut-off manufacturer), 48.9 and 95.4 corresponded to a specificity of $\sim 85.0 \%, \sim 95 \%$, and $\sim 97.5 \%$. The LR (95\% CI) for AARD was 0.160 (CI 0.128-0.200), 1.285 (0.865-1.907), 3.280 (1.918-5.608 and 27.921 (17.838-43.704) for test results (CU) $<20.00$, $20.00-48.90,48.90-95.40$ and $\geq 95.40$, respectively. The fraction of patients with a test result in each of these test result intervals was $14.2 \%$, $8.3 \%, 8.1 \%$, and $69.4 \%$, respectively. Thus, for values $>48.9$, the LR for AARD was $>1 ; 85.8 \%$ of the AARD patients had such result. $69 \%$ of patients had a test result with a LR $>20$.
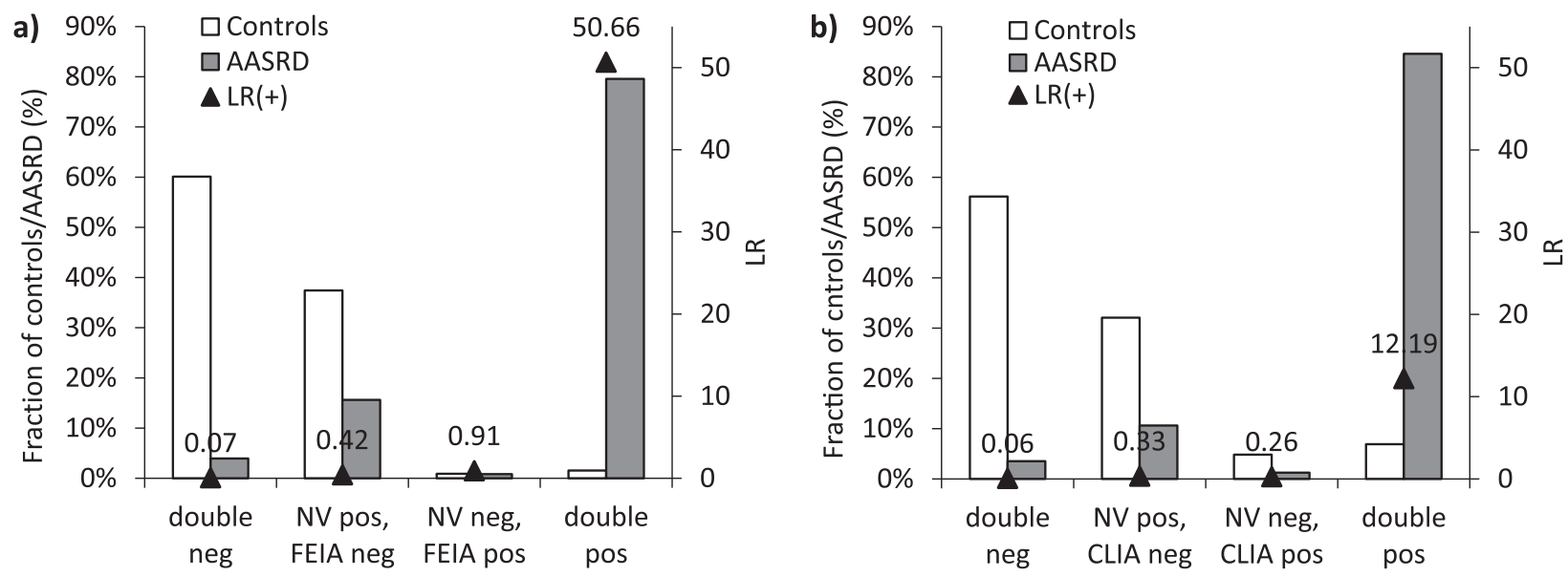

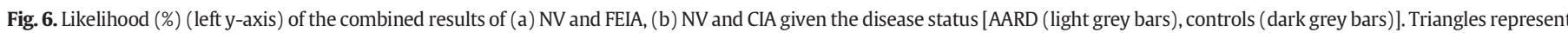
LR (second y-axis). 
Table 2

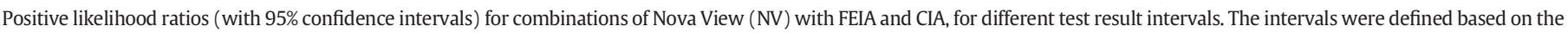
cut-off prescribed by the manufacturer and on thresholds corresponding to predefined specificities: $<85.0 \%, 85.0-95.0 \%, 95.0-97.5 \%, 97.5-100.0 \%$ and $100.0 \%$.

\begin{tabular}{|c|c|c|c|c|c|c|c|}
\hline & & \multicolumn{6}{|l|}{ NV (LIU) } \\
\hline & & $\leq 48$ & $48-158$ & $158-522$ & $522-910$ & $910-3382$ & $>3382$ \\
\hline \multirow[t]{5}{*}{ FEIA (ratio) } & $\leq 0.40$ & $0.06(0.04-0.1)$ & $0.09(0.04-0.2)$ & $0.5(0.3-0.8)$ & $0.3(0.0-1.9)$ & $2.5(1.2-5.3)$ & $\infty(0.79-\infty)$ \\
\hline & $0.40-0.70$ & $0.2(0.0-0.6)$ & $0.2(0.0-0.7)$ & $0.5(0.1-1.8)$ & $2.4(0.5-\infty)$ & $4.0(0.8-\infty)$ & n.d. \\
\hline & $0.70-1.00$ & n.d. & $0.5(0.2-3.0)$ & $3.2(0.6-\infty)$ & $1.6(0.5-\infty)$ & $6.4(1.0-\infty)$ & n.d. \\
\hline & $1.00-8.60$ & $0.9(0.2-3.6)$ & $3.6(1.3-\infty)$ & $8.3(3.6-35.6)$ & $\infty(19.1-\infty)$ & $68.7(28.5-\infty)$ & $\infty(23.6-\infty)$ \\
\hline & $>8.60$ & n.d. & $\infty(0.5-\infty)$ & $\infty(0.9-\infty)$ & $\infty(10.2-\infty)$ & $\infty(52.6-\infty)$ & $\infty(11.3-\infty)$ \\
\hline \multirow[t]{4}{*}{$\mathrm{CIA}(\mathrm{CU})$} & $<20.0$ & $0.06(0.04-0.1)$ & $0.1(0.1-0.2)$ & $0.5(0.3-0.8)$ & $0.5(0.1-1.3)$ & $2.7(1.2-7.3)$ & $\infty(0.4-\infty)$ \\
\hline & $20.0-48.90$ & $0.3(0.1-0.7)$ & $0.3(0.0-0.9)$ & $1.8(0.7-4.5)$ & $4.0(0.8-\infty)$ & $25.6(6.0-\infty)$ & $\infty(0.4-\infty)$ \\
\hline & $48.90-95.40$ & $0.2(0.1-0.6)$ & $1.6(0.4-\infty)$ & $4.8(0.9-\infty)$ & $4.8(1.4-\infty)$ & $13.6(4.2-\infty)$ & $\infty(0.5-\infty)$ \\
\hline & $\geq 95.40$ & $0.3(0.1-0.8)$ & $2.7(0.6)$ & $9.6(3.4-\infty)$ & $\infty(24.3-\infty)$ & $46.3(24.8-128.0)$ & $\infty(35.8-\infty)$ \\
\hline
\end{tabular}

n.d. 'not determined', LR could not be calculated because of lack of samples in this category.

\subsection{Combining IIF with SPA}

Fig. 1 shows FEIA (ratio) and CIA (CU) test results as a function of NV LIU. Sensitivity and specificity for double positivity was $79.6 \%(75.3 \%-$ 82.7\%) and 98.4\% (97.3\%-99.2\%) for NV combined with FEIA and 84.6\% (81.1\%-87.7\%) and 93.1\% (91.1\%-94.8\%) for NV combined with CIA.

The LR $(95 \% \mathrm{CI})$ for double positivity, double negativity, single positivity NV and single positivity SPA was, respectively 50.7 (30.8-100.6), 0.07 (0.04-0.1), $0.42(0.3-0.5)$, and $0.91(0.2-3.6)$ for NV combined with FEIA and 12.2 (9.5-16.2), 0.06 (0.04-0.1), 0.33 (0.2-0.4), 0.26 (0.08-0.6) for NV combined with CIA, respectively (Fig. 6). Thus, double positivity of NV and a SPA increased the LR from 2.4, 32.5 and 7.3 for, respectively, NV, FEIA and CIA alone, to 50.7 for the combination of NV with FEIA and 12.2 for the combination of NV with CIA. The fraction of patients and controls (likelihoods) for different combinations of test results of NV and FEIA or CIA are also shown in Fig. 5.

Finally, the LR not only increased with double positivity, but also with increasing antibody levels (Table 2). For example, FEIA results $>1$ ratio combined with NV LIU $>522$ [LR $\infty$ ] or CIA result $\geq 95.4$ CU together with NV LIU $>522[$ LR $\infty$ ] strongly suggested the presence of an AARD.

\section{Discussion}

During the last decade, the landscape of laboratory screening for AARD has remarkably changed by the introduction of automated SPAs and IIF systems that allow computerized interpretation of fluorescence patterns and intensity $[3,4,6-8,14,16,17]$. We here report on the performance of one automated HEp-2 IIF and two automated SPA-based screening assays (FEIA and CIA) evaluated on a large cohort of diagnostic samples from AARD patients and controls.

Applying the cut-off proposed by the manufacturer revealed marked differences in sensitivity and specificity among assays, with the highest (97.5\%) and lowest (61\%) specificity found for FEIA and NV, respectively. A similar low (64.7\%) specificity for NV was reported by Bentow et al. who studied 178 AARD patients and 204 controls [14]. The specificity of CIA (88.3\%) (85.5\%-90.4\%) in our study was lower than the specificity reported by Bentow et al. (94.1\%; 95\% CI: 90.0-96.9\%)) [14]. The sensitivity was highest for NV (95\%), followed by CIA (86\%) and FEIA (80\%). The sensitivities of NV and CIA in our study were higher than the sensitivities ( $84.8 \%$ and $78.1 \%$, respectively) reported by Bentow et al. [14]. This might be related to the composition of the study population (our study included diagnostic samples from untreated patients). For NV, the LR in our study was similar to the LR reported by Bentow et al. [14].

When all AARD were combined, the AUC was similar for FEIA, CIA and NV. Thus, differences in sensitivity and specificity between the methods are related to differences in the way cut-off values have been set by the manufacturers, although, other factors may contribute as well. Breakdown of the AARD group revealed disease-dependent differences in AUC. This was most pronounced for SjS, for which the AUC was significantly higher for the SPAs than for NV. This is related to the better detection of SSA-/Ro antibodies by SPA than by HEp-2 cell IIF. When partial AUCs were calculated for high specificity thresholds ( $\geq 90 \%)$, then differences in PAUC were observed between FEIA and CIA. Finally, the antigen composition of SPA has a likely impact on performance variability between the different platforms, as illustrated for SSc-related antibodies.

Since autoantibodies are known to precede the clinical onset of AARD [18], it is of interest to study the long-term evolution of patients with discrepant results between SPA and IIF. Recently, it was demonstrated that a significant number of patients positive for ANA by SPA and negative by IIF at baseline developed ANA by IIF as well as clinically defined AARD during follow-up [19]. An important aspect in the difference between ANA by IIF vs. SPA is the presence of anti-DFS70 antibodies which is a common cause of clinically false positive cases by IIF on HEp-2 cells $[20,21]$. Although this likely contributed to the difference between IIF and SPA in our study, we did not test for anti-DFS70 antibodies in the discrepant samples.

In order to improve interpretation of a specific test result, we calculated test result interval-specific LR. The intervals were delimited by thresholds that corresponded to predefined specificities. Such an approach allows a refined estimation of the $L R$, which is not possible by applying a single cut-off. The LR for AARD increased with increasing antibody level for all assays studied.

IIF is considered the reference method of choice for ANA detection in AARD because of its high sensitivity [16,22]. The drawback is the low specificity and corresponding low LR. In our study, $11 \%$ of AARD patients had a positive IIF result that was associated with a LR of 1 and $5 \%$ of AARD patients had a positive IIF result that was associated with a LR of 0.2 . This illustrates that $16 \%$ of AARD patients had a low positive IIF result with a low LR. By contrast, a test result that corresponded to a LR $>20$ was found in $66 \%, 69 \%$ and $80 \%$ of the patients for IIF, CIA and FEIA, respectively. If the cut-off of IIF and SPA was set at a value that corresponded to a specificity of 95\%, then the sensitivity (and LR) of SPAs equalled or exceeded the sensitivity of NV, although differences between the two SPAs were observed. Of note, at such cutoff level, sensitivity for IIM was lower for IIF than for SPA, even though many antigens associated with IIM are missing in SPAs. This is related to the fact that IIF reactivity in IIM is lower than IIF reactivity in other AARD patients. Moreover, a fraction of the antigens in IIM are located in the cytoplasm, which are known to exhibit limited sensitivity in IIF [16].

IIF provides information on the pattern, which can give additional information, e.g. on disease association. Such information is lacking from SPAs.

The LR and specificity not only increased for high antibody levels but also for double positivity of IIF and SPA. Previous studies have reported similar observations [23]. Double negativity (IIF and SPA) was associated with a low $\operatorname{LR}(<0.1)$. In our cohort, $3.5 \%$ of AARD patients were 
double negative. From a clinical perspective, our data support the parallel use of IIF and SPA. The drawback of combining two assays is the higher cost. However, parallel testing might reduce costs for unnecessary referrals and improve referral times for urgent patients. Health economic studies are desired to address this aspect.

The strength of this study is the large multicentre cohort of diagnostic samples from AARD patients $(n=480)$ used for evaluating assay performance. Our results indicate differences in performance between different assays, which was disease-dependent. Taking into account antibody levels and combining IIF with SPA adds value.

\section{References}

[1] Tozzoli R, Bizzaro N, Tonutti E, Villalta D, Bassetti D, Manoni F, et al. Guidelines for the laboratory use of autoantibody tests in the diagnosis and monitoring of autoimmune rheumatic diseases. Am J Clin Pathol 2002;117:316-24.

[2] Agmon-Levin N, Damoiseaux J, Kallenberg C, Sack U, Witte T, Herold M, et al. International recommendations for the assessment of autoantibodies to cellular antigens referred to as anti-nuclear antibodies. Ann Rheum Dis 2014;73:17-23.

[3] Op De Beeck K, Vermeersch P, Verschueren P, Westhovens R, Mariën G, Blockmans $\mathrm{D}$, et al. Detection of antinuclear antibodies by indirect immunofluorescence and by solid phase assay. Autoimmun Rev 2011;10:801-8.

[4] de Almeida Brito F, Maria Elói Santos S, Aparecida Ferreira G, Pedrosa W, Gradisse J, Cristina Costa L, et al. Diagnostic evaluation of ELISA and chemiluminescent assays as alternative screening tests to indirect immunofluorescence for the detection of antibodies to cellular antigens. Am J Clin Pathol 2016;145:323-31.

[5] Agmon-Levin N, Damoiseaux J, Shoenfeld Y. Response to: 'Detection of antinuclear antibodies: added-value of solid phase assay?' by Bossuyt and Fieuws. Ann Rheum Dis 2014;73:e11.

[6] Schouwers S, Bonnet M, Verschueren P, Westhovens R, Blockmans D, Mariën G, et al. Value-added reporting of antinuclear antibody testing by automated indirect immunofluorescence analysis. Clin Chem Lab Med 2014;52:547-51.

[7] Oyaert M, Bossuyt X, Ravelingien I, Van Hoovels L. Added value of indirect immunofluorescence intensity of automated antinuclear antibody testing in a secondary hospital setting. Clin Chem Lab Med 2016;54:e63-6.

[8] Bizzaro N, Antico A, Platzgummer S, Tonutti E, Bassetti D, Pesente F, et al. Automated antinuclear immunofluorescence antibody screening: a comparative study of six computer-aided diagnostic systems. Autoimmun Rev 2014;13:292-8.

[9] Hochberg MC. Updating the American College of Rheumatology revised criteria for the classification of systemic lupus erythematosus. Arthritis Rheum 1997;40:1725.
[10] van den Hoogen F, Khanna D, Fransen J, Johnson SR, Baron M, Tyndall A, et al. Classification criteria for systemic sclerosis: an American college of rheumatology/ European league against rheumatism collaborative initiative. Ann Rheum Dis 2013;72:1747-55 (2013).

[11] Shiboski SC, Shiboski CH, Criswell L, Baer A, Challacombe S, Lanfranchi H, et al. American College of Rheumatology classification criteria for Sjögren's syndrome: a data-driven, expert consensus approach in the Sjögren's International Collaborative Clinical Alliance cohort. Arthritis Care Res 2012;64:475-87.

[12] Bohan A, Peter JB, Bowman RL, Pearson CM. Computer-assisted analysis of 153 patients with polymyositis and dermatomyositis. Medicine (Baltimore) 1977;56: 255-86.

[13] Alarcón-Segovia D, Cardiel MH. Comparison between 3 diagnostic criteria for mixed connective tissue disease. Study of 593 patients. J Rheumatol 1989;16:328-34.

[14] Bentow C, Lakos G, Rosenblum R, Bryant C, Seaman A, Mahler M. Clinical performance evaluation of a novel, automated chemiluminescent immunoassay, QUANTA Flash CTD Screen Plus. Immunol Res 2015;61:110-6.

[15] Bossuyt X. Clinical performance characteristics of a laboratory test. A practical approach in the autoimmune laboratory. Autoimmun Rev 2009;8:543-8.

[16] Mahler M, Meroni PL, Bossuyt X, Fritzler MJ. Current concepts and future directions for the assessment of autoantibodies to cellular antigens referred to as anti-nuclear antibodies. J Immunol Res 2014;2014:315179.

[17] Op De Beéck K, Vermeersch P, Verschueren P, Westhovens R, Mariën G, Blockman $\mathrm{D}$, et al. Antinuclear antibody detection by automated multiplex immunoassay in untreated patients at the time of diagnosis. Autoimmun Rev 2012;12:137-43.

[18] Arbuckle MR, McClain MT, Rubertone MV, Scofield RH, Dennis GJ, James JA, et al. Development of autoantibodies before the clinical onset of systemic lupus erythematosus. N Engl J Med 2003;349:1526-33.

[19] Pérez D, Gilburd B, Cabrera-Marante Ó, Martínez-Flores JA, Serrano M, Naranjo L, et al. Predictive autoimmunity using autoantibodies: screening for anti-nuclear antibodies. Clin Chem Lab Med 2017 Jun 17. https://doi.org/10.1515/cclm-2017-0241 [Epub ahead of print]

[20] Conrad K, Röber N, Andrade LE, Mahler M. The clinical relevance of anti-DFS70 autoantibodies. Clin Rev Allergy Immunol 2017;52:202-16.

[21] Mahler M, Meroni PL, Andrade LE, Khamashta M, Bizzaro N, Casiano CA, et al. Towards a better understanding of the clinical association of anti-DFS70 autoantibodies. Autoimmun Rev 2016;15:198-201.

[22] Meroni PL, Schur PH. ANA screening: an old test with new recommendations. Ann Rheum Dis 2010;69:1420-2.

[23] Bossuyt X, Fieuws S. Detection of antinuclear antibodies: added value of solid phase assay? Ann Rheum Dis 2014;73:e10. 